This is a preprint version of Hervella et al. Curr. Top. Med. Chem. 14, 115-1123, 2014

The original article can be found at:

http://benthamscience.com/journal/abstracts.php?journalID=ctmc\&articleID=121253

\title{
PEGYLATED LIPID NANOCAPSULES WITH IMPROVED DRUG ENCAPSULATION AND CONTROLLED RELEASE PROPERTIES
}

Pablo Hervella ${ }^{1}$, Maria Alonso-Sande ${ }^{1}$, Francisco Ledo ${ }^{2}$, Maria L. Lucero ${ }^{2}$, Maria J. Alonso $^{1}$, Marcos Garcia-Fuentes ${ }^{1 *}$

${ }^{1}$ Center for Research in Molecular Medicine and Chronic Diseases (CIMUS), Department of Pharmacy and Pharmaceutical Technology and Health Research Institute of Santiago de Compostela (IDIS), Campus Vida, Universidad de Santiago de Compostela, Spain.

${ }^{2}$ FAES FARMA S.A. R\&D and Innovation Department. Autonomía n ${ }^{\circ} 10$, Leioa, Bizkaia, Spain.

*Corresponding author: Prof. Marcos Garcia-Fuentes

Ed. CIMUS T6D1, Av. Barcelona s/n

Campus Vida, Universidad de Santiago de Compostela

15782 Santiago de Compostela, Spain

TLF: +34881815450

www.enthuse.me/marcosgf

e-mail: marcos.garcia@usc.es 


\begin{abstract}
Drugs with poor lipid and water solubility are some of the most challenging to formulate in nanocarriers, typically resulting in low encapsulation efficiencies and uncontrolled release profiles. PEGylated nanocapsules (PEG-NC) are known for their amenability to diverse modifications that allow the formation of domains with different physicochemical properties, an interesting feature to address a drug encapsulation problem. We explored this problem by encapsulating in PEG-NC the promising anticancer drug candidate F10320GD1, used herein as a model for compounds with such characteristics. The nanocarriers were prepared from Miglyol ${ }^{\circledR}$, lecithin and PEG-sterate through a solvent displacement technique. The resulting system was a homogeneous suspension of particles with size around $200 \mathrm{~nm}$. F10320GD1 encapsulation was found to be very poor $(<15 \%)$ if PEG-NC were prepared using water as continuous phase; but we were able to improve this value to $85 \%$ by fixing the $\mathrm{pH}$ of the continuous phase to 9 . Interestingly, this modification also improved the controlled release properties and the chemical stability of the formulation during storage. These differences in pharmaceutical properties together with physicochemical data suggest that the $\mathrm{pH}$ of the continuous phase used for PEG-NC preparation can modify drug allocation, from the external shell towards the inner lipid core of the nanocapsules. Finally, we tested the bioactivity of the drug-loaded PEG-NC in several tumor cell lines, and also in endothelial cells. The results indicated that drug encapsulation led to an improvement on drug cytotoxicity in tumor cells, but not in non-tumor endothelial cells. Altogether, the data confirms that PEG-NC show adequate delivery properties for F10320GD1, and underlines its possible utility as an anticancer therapy.
\end{abstract}

\title{
Graphical Abstract:
}

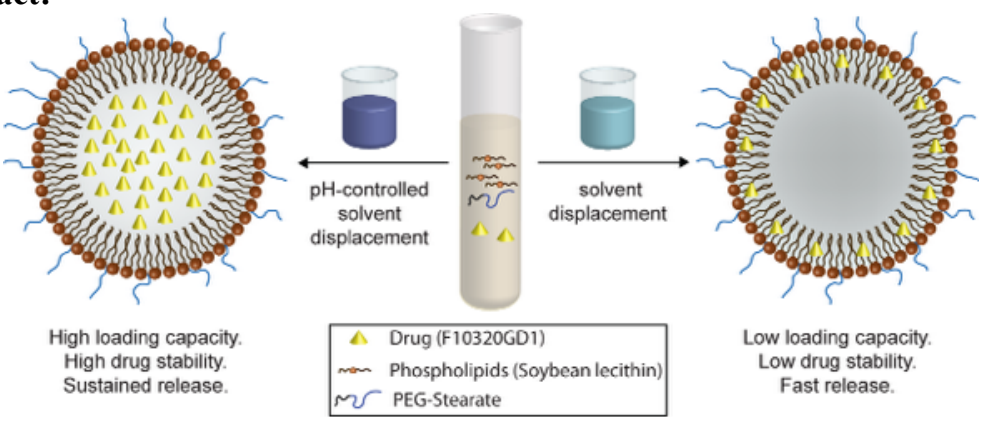

Keywords: nanocapsules, nanoemulsions, formulation, encapsulation, controlled release, PEG, cancer. 


\section{INTRODUCTION}

Effective drug encapsulation and controlled release are key properties of drug nanocarriers. It has been frequently stated that nanocarriers can impart beneficial biopharmaceutical properties to active molecules, including enhanced solubility, higher cellular and tissue penetration, reduced side effects, and improved biodistribution [1-5]. However, premature release of the drug from the nanocarrier, a most frequent situation, would ruin any of these benefits [6]. To be effective, nanocarriers need to be engineered to ensure tight integration between drugs and nanocarriers, and to attain drug release with appropriate kinetics.

A particularly challenging type of drugs for formulation in nanocarriers are those with modest solubility both in oils and in water. Due to the limited affinity of the drug for the different domains of the nanocarriers, these compounds will usually present poor encapsulation and uncontrolled burst release [7-8]. This has been observed for a variety of nanocarriers including liposomes, nanoemulsions, and polymeric na ${ }^{-}$ noparticles [9-12].

PEGylated nanocapsules (PEG-NC) are colloidal drug delivery systems characterized by an oily core and a highly structured PEG-lipid shell. We hypothesized that PEG-NC could be useful for formulating challenging drugs such as those described above due to the possibility to optimize drug-oil affinity by technological approaches [13]. Besides, PEG-NC are excellent nanocarriers for intravenous drug administration: they can be prepared using excipients with an acceptable regulatory status, and through industryfriendly procedures. The nanocapsules' PEG-coating endows the system with adequate stability in physiological media, and eventually, with long-circulation properties upon intravenous administration that are beneficial for passive targeting to tumoral and inflamed regions [14-15].

We have previously shown that the incorporation of PEG-stearate (PEG-STE, Symulsol ${ }^{\circledR}$ M52) to lipid formulations leads to the formation of dense polymeric coatings, above 2 chains $/ \mathrm{nm}^{2}$, that are ideal for protein repulsion and stealth properties [16-18]. Moreover, PEG-STE may form phase-separated solid lipid layers on the surface of the nanocapsules, which could act as a diffusional barrier to prevent drug leakage. Indeed,we have previously measured the diffusion coefficients of PEG-STE coated nanoparticles by NMR and we have found that while the diffusion coefficient of the particles was $9,9 \times 10^{-13} \mathrm{~m}^{2} \mathrm{~s}^{-1}$ (corresponding to the Brownian motion of a sphere of $200 \mathrm{~nm}$, the size of the nanoparticle), the observed diffusion coefficient for the oil inside the particle was 35 times higher and similar to the pure oil $\left(3 \times 10^{-11} \mathrm{~m}^{2} \mathrm{~s}^{-1}\right)$, indicating the confinement of the oil inside the protective PEG-STE monolayer [19]. We hypothesized that the presence of a solid surfactant shell on the surface could be useful to impart controlled release to PEG-NC loaded with drugs with modest solubility in water and oils, providing we were able to integrate the drug in the lipid core of the nanocarrier. 
In this manuscript, we report the design of PEG-NC for the delivery of F10320GD1, an anticancer drug candidate used herein as a model compound exhibiting modest solubility in water and in oils. In order to improve the encapsulation of F10320GD1 into PEG-NC, we fixed the $\mathrm{pH}$ of the continuous aqueous phase during nanocarrier preparation, a strategy previously used with other systems [9, 20-21]. Our results indicate that this modification leads to important changes in the characteristics of the formulation that cannot be attributed to a simple shift in partition equilibria, but rather to modifications in the structure of the nanocarrier-drug interaction. This formulation strategy could be potentially extended for encapsulating other drugs with similar characteristics in structured lipid nanocarriers.

\section{MATERIALS AND METHODS}

\subsection{Materials}

Soybean lecithin (EPIKURON® 170) was donated by Cargill (Spain). Miglyol® 812 (caprylic and capryc fatty acid esters) was donated by Sasol Germany GmbH (Germany). Polyethylene glycol stearate (PEG-STE, Symulsol ${ }^{\circledR}$ M52, MW=1750 Da) was donated by Seppic (France). Poloxamer 188 (Pluronic ${ }^{\circledR}$ F68) was purchased by Sigma-Aldrich, Spain. The anticancer drug candidate F10320D1 was provided by FAES FARMA S.A. (Spain).

\subsection{Preparation of PEGylated nanocapsules}

PEGylated nanocapsules (PEG-NC) were prepared by a solvent displacement method [22]. For these systems, $25 \mathrm{mg}$ of lecithin were dissolved in $10 \mathrm{~mL}$ of a acetone:ethanol $(10: 1 \mathrm{v}: \mathrm{v})$ mixture in presence of variable amounts $(0,14,34$ or $68 \mu \mathrm{mol})$ of polyethylene glycol stearate (PEG-STE) and $0.125 \mathrm{ml}$ of Miglyol ${ }^{\circledR}$. This organic phase was poured into $20 \mathrm{~mL}$ of an aqueous phase composed of poloxamer 188 $(0.25 \% \mathrm{w} / \mathrm{v})$ under stirring, leading to the immediate formation of a milky dispersion. Finally, the organic solvents were removed under vacuum, and the PEG-NC were concentrated to a fixed volume of $10 \mathrm{~mL}$.

Drug loaded PEG-NC were prepared by the method described above, but adding the drug to the organic phase together with the oil and the surfactants. PEG-NC prepared with controlled $\mathrm{pH}$ were prepared by the same method, but the continuous aqueous phase contained both poloxamer 188 and a sodium carbonate $\left(\mathrm{Na}_{2} \mathrm{CO}_{3}\right)$ buffer adjusted to $\mathrm{pH} 9$.

\subsection{Physico-chemical characterization of PEG-NC}

The particle size and polydispersity index of PEG-NC were measured by photon correlation spectroscopy 
(PCS). Samples were diluted in MilliQ water and the analysis was performed at fixed temperature with an angle detection of $90^{\circ}$. Z-potential was calculated from the mean electrophoretic mobility values determined by laser Doppler anemometry (LDA). Samples were diluted with potassium chloride (KCl) $1 \mathrm{mM}$ and placed in an electrophoretic cell where a potential of $\pm 150 \mathrm{mV}$ was applied. PCS and LDA analysis were performed using a Zetasizer 3000HS (Malvern Instruments, Malvern, U.K.). Each sample was analyzed in triplicate.

The morphological examination of the PEG-NC was performed by transmission electron microscopy (TEM, CM12 Philips, Netherland). Samples were stained with sodium phosphotungstic acid 2\% (w/v), and placed on copper grids with Formvard ${ }^{\circledR}$ for visualization.

The degree of PEGylation of the nanocapsules was assessed by ${ }^{1} \mathrm{H}-\mathrm{NMR}$, after isolation by ultracentrifugation of PEG-NC from non-associated PEG-STE (27400 g, 1 hour, $\left.4{ }^{\circ} \mathrm{C}\right)$ [16]. After isolation, the samples were diluted in deuterium oxide $\left(\mathrm{D}_{2} \mathrm{O}\right)$ and analyzed in a Bruker DRX 500 spectrophotometer.

\subsection{Encapsulation efficiency (E.E.) of loaded PEG-NC}

The encapsulation efficiency of F10320GD1 in PEG-NC was determined by calculating the difference between the total amount of drug present in the formulation and the free drug found in the supernatant of the formulation after centrifugation. The total concentration of drug in the formulation was measured by dissolving an aliquot in acetonitrile and analyzing the drug content by high-performance liquid chromatography (HPLC). The free drug concentration was measured by the same method following separation from the encapsulated drug by ultracentrifugation $\left(27400 \mathrm{~g}, 1\right.$ hour, $\left.4{ }^{\circ} \mathrm{C}\right)$. F10320GD1 quantification by HPLC was performed according to an analytical method provided by FAES FARMA S. A.

\subsection{Stability studies}

The stability of blank PEG-NC was evaluated under storage for 90 days at $4^{\circ} \mathrm{C}, 25^{\circ} \mathrm{C}$ and $37^{\circ} \mathrm{C}$. Particle size, polydispersity index (P.I.) and Z-potential was assessed at different time points and measured as described above. Drug concentration and encapsulation efficiency of drug loaded PEG-NC was also evaluated under storage conditions for 30 days at $4^{\circ} \mathrm{C}$ using the methods described above.

The stability of blank PEG-NC was also evaluated after 24 hours incubation at $37^{\circ} \mathrm{C}$ in different aqueous solutions: saline solution $0.9 \%(\mathrm{w} / \mathrm{v})$, supplemented medium DMEM+, PBS and water. Size and polydispersity index were measured after 24 hours as described above. 


\subsection{In vitro release studies}

Release studies were carried out by incubating loaded PEG-NC in PBS at an appropriate concentration to ensure sink conditions. The samples were placed in an incubator at $37^{\circ} \mathrm{C}$ with horizontal shaking and collected at different time intervals. The percentage of drug released was quantified by HPLC after separation of the encapsulated drug by ultracentrifugation as described in 2.4.

\subsection{Cell culture}

The cytotoxic effect of the free and encapsulated drug was evaluated in four different human cell cultures. Three human cell lines were used, MCF7, SF268, and NCI-H460, models for breast, glioblastoma and lung cancer, respectively. The cytotoxicity was also tested in a primary culture of human umbilical vein endothelial cells (HUVEC). The human cell lines used in this study were obtained from the American Type Culture Collection (ATCC), and they were cultured in RPMI-1640 medium containing 10\% (v/v) heat-inactivated fetal bovine serum, $2 \mathrm{mM} \mathrm{L}$-glutamine, $100 \mathrm{U} / \mathrm{ml}$ penicillin, and $100 \mu \mathrm{g} / \mathrm{ml}$ streptomycin at $37^{\circ} \mathrm{C}$ in humidified $95 \%$ air and $5 \% \mathrm{CO}_{2}$. HUVEC were obtained by collagenase digestion of umbilical cord veins as described elsewhere [23], and cultured under the same conditions.

\subsection{Cell viability assay}

Cell viability was determined by an XTT colorimetric method. Known amounts of exponentially growing cells were seeded in 96-well flat-bottomed microtiter plates, at a final volume of $100 \mu$ l. Seeded cells were incubated for $24 \mathrm{~h}$ at $37^{\circ} \mathrm{C}$, in a humidified atmosphere of $5 \% \mathrm{CO}_{2} / 95 \%$ air to allow cell attachment to the plates. Then, cells were incubated for $72 \mathrm{~h}$ with different concentrations of the tested formulations or controls, under standard culture conditions. Cell proliferation was quantified using the XTT (3'-[1(phenylamino)carbonyl]-3,4-tetrazolium-bis(4-methoxy-6-nitro)benzene sulfonic acid sodium salt hydrate) cell proliferation kit (Roche Molecular Biochemicals, Mannheim, Germany) following the manufacturer's instructions. Briefly, a freshly prepared mixture solution $(50 \mu \mathrm{l})$ of XTT labeling reagent and PMS ( $N$-methyldibenzopyrazine methyl sulfate) electron coupling reagent was added to each well. The resulting mixtures were incubated for $4 \mathrm{~h}$ in a humidified atmosphere $\left(37^{\circ} \mathrm{C}, 5 \% \mathrm{CO}_{2}\right)$, and the absorbance of the formazan product generated was measured in a microtiter plate reader at a test wavelength of $490 \mathrm{~nm}$ and a reference wavelength of $655 \mathrm{~nm}$. The $\mathrm{IC}_{50}(50 \%$ inhibitory concentration) was calculated as the drug concentration causing a 50\% inhibition in cell proliferation. Data is represented as mean values of four independent experiments each performed in triplicates. The drug concentrations tested were $20,10,5,1,0.1 \mu \mathrm{M}$. 


\subsection{Statistical analysis}

All statistical analyses were performed using the software OriginPro (Northampton, MA, US). Experiments were analyzed using a one-way analysis of variance (ANOVA) followed by Tukey's test to determine the significance of all paired combinations. Differences were considered to be significant at a level of $\mathrm{p}<0.05$.

\section{RESULTS \& DISCUSSION}

In this work, we have developed a nanotechnology formulation strategy that enables the encapsulation of drugs with poor solubility both in water and in oils, and its controlled release from the nanocarriers. The nanocarriers, PEGylated nanocapsules (PEG-NC), consist on an oily core stabilized with a PEG-stearate (PEG-STE) shell. To address this formulation challenge, a promising anticancer drug candidate, F10320GD1, was used as a model for compounds with these physicochemical properties. The developed PEG-NC loaded with F10320GD1 were characterized with respect to their size, zeta potential, stability, encapsulation efficiency and controlled release properties. Finally selected prototypes were selected for the evaluation of their activity in cell cultures.

\subsection{Preparation and characterization of PEG-NC}

We designed PEG-NC based on a triglyceride oil (Miglyol ${ }^{\circledR} 812$ ), and a tensoactive (lecithin), materials with known utility for the encapsulation of hydrophobic and amphipilic drugs, and suitable for i.v. injection [24-25]. We also incorporated a protective PEG coating around the nanostructure by including PEGSTE as an additional component. The use of PEG-linked lipids has been previously described as an effective strategy to PEGylate lipid systems that avoids chemical conjugation and the use of costly PEGylated phospholipids [26-28]. PEG-NC were prepared following a solvent displacement technique, a simple and mild method that is easily scalable. PEG-NC formation was assessed by transmission electron microscopy (TEM) and photon correlation spectroscopy (PCS) measurements.

In a first step, we aimed at studying the amount of PEG-STE necessary to achieve a suitable surfactant shell. Previous studies from our group have shown that lipid nanocarriers can be coated with PEG by the addition of PEG-STE, achieving polymer densities that should be suitable for stealth nanocarriers [16]. In this work, we studied different proportions of PEG-STE in the lipid mixture $(0-60 \% \mathrm{w} / \mathrm{w})$, and analyzed its effect on nanocarrier structure and morphology, particle size, and Z-potential.

TEM images indicated that PEG-NC are homogeneous, with spherical morphology, and particle size around $200 \mathrm{~nm}$ (Figure 1). The presence of different amounts of PEG-STE affected neither the size nor 
the morphology of PEG-NC, but at $60 \%(\mathrm{w} / \mathrm{w})$ PEG-STE another population of small particles appeared in the TEM images. This second population can be attributed to the formation of PEG-STE micelles once the PEG-NC shell is saturated.

The results obtained by TEM were confirmed by dynamic light scattering (Table 1). All PEG-NC showed a low polydispersity index (P.I.) (below 0.2 in all cases) and a mean size between $150-200 \mathrm{~nm}$. The inclusion of the non-ionic polymer PEG-STE in the formulation did not significantly affect the hydrodynamic diameter of PEG-NC, however, a neutralization of the Z-potential value (from - 38 to $-22 \mathrm{mV}$ ) was noted as the amount of PEG-STE was increased to $30-60 \%(\mathrm{w} / \mathrm{w})$. The variation of the Z-potential with the content of PEG-STE can be attributed to a shielding effect of PEG on the nanocapsule surface, and is a typical observation for PEGylated colloidal systems. Based on the fact that further reductions in Z-potential were not observed between 30 and $60 \%(\mathrm{w} / \mathrm{w})$, and on the observation of a second population of micellar structures for the highest PEG-STE proportion (see above), we decided to fix this parameter for future studies at $30 \%(\mathrm{w} / \mathrm{w})$.

NMR studies performed on isolated PEG-NC confirmed the PEGylation on the nanocapsules. PEG-NC were washed by centrifugation to remove the non-bound PEG-STE and then analyzed by ${ }^{1} \mathrm{H}-\mathrm{NMR}$. A well resolved peak was observed at $3.6 \mathrm{ppm}$, thus confirming the presence of PEG on the formulation [16] (Supplementary material, Figure S1).

The stability of PEG-NC was tested in different media upon incubation at $37^{\circ} \mathrm{C}$ for 24 hours (Figure 2). PEG-NC showed good stability both in water and in supplemented medium (D-MEM). However, in solutions with higher ionic strength such as saline solution or PBS, particle aggregation was observed. To increase the stability of PEG-NC in these media, poloxamer 188 was included in the formulation. The results showed that the addition of poloxamer 188 did not alter the size or the Z-potential of the resulting PEG-NC, however their stability in PBS and saline solution was significantly increased. Indeed, no aggregation was observed after 24 hours at $37^{\circ} \mathrm{C}$ in these media. Steric stabilization of nanocapsules by nonionic surfactants, has been previously described, and it is considered essential to preserve the colloidal characteristics in physiologically fluids [29]. In this work, PEG-STE and poloxamer 188 seem to work cooperatively to achieve an optimal colloidal stability in buffered media with ionic strength and $\mathrm{pH}$ similar to those of plasma.

Finally, we tested the stability of PEG-NC upon storage for three months at different temperatures $(4,25$ and $37^{\circ} \mathrm{C}$ ). This stability was assessed by measuring the size and P.I. of PEG-NC at different times during this incubation period. Neither particle aggregation nor size increase due to Ostwald ripening was observed for up to 90 days, not even at $37^{\circ} \mathrm{C}$ (Figure 3).

Based on all this characterization, the final composition of PEG-NC used throughout this work was selected: Miglyol ${ }^{\circledR} 55 \% \mathrm{w} / \mathrm{w}$, lecithin $12 \% \mathrm{w} / \mathrm{w}$, PEG-STE $28 \% \mathrm{w} / \mathrm{w}$ and poloxamer $5 \% \mathrm{w} / \mathrm{w}$. 


\subsection{Encapsulation of F10320D1 in PEG-NC}

Hydrophilic macromolecules can be encapsulated in liposomes and in some polymeric nanoparticles with relatively high efficacy [30-32], whereas highly hydrophobic drugs are good candidates to be entrapped in lipid-based formulations (liposomes, nanocapsules, lipid nanoparticles, nanoemulsions) [33-34]. However, drugs with limited solubility both in lipid an aqueous media, often prove difficult to encapsulate in nanocarriers.

We have previously described several modifications that can be applied to the core and the shell of lipid nanocarriers $[16,19,26]$. Based on this structural flexibility of lipid nanocarriers, we proposed the potential interest of PEG-NC as delivery systems for drugs with poor solubility both in water and in oils. Besides these considerations, PEG-NC offer other additional advantages considering future applications for the model cytotoxic drug used in this study: F10320D1. Namely, their dense PEG shell will allow these nanocarriers to circulate for a prolonged time in the bloodstream and accumulate in solid tumors by passive targeting [35-36].

F10320D1 has the following physicochemical characteristics: low molecular weight $(\sim 450 \mathrm{Da}), \mathrm{pKa}$ between $8-9$, low solubility in water $(0.5 \mathrm{mg} / \mathrm{mL}, 1 \mathrm{mM})$ and also in oils. PEG-NC formation proceeded normally in the presence of the drug, resulting in the instantaneous formation of a homogeneous colloidal systems. Unfortunately, when we prepared PEG-NC with loadings suitable to reach final drug concentrations optimal for in vivo experiments $(2 \mathrm{mg} / \mathrm{mL}, 4 \mathrm{mM})$, the encapsulation efficiency (E.E.) was unacceptable $(15 \%)$. The low EE value obtained was attributed to the low solubility of the drug in the oily phase that forces drug allocation towards the negatively charged PEG-NC surface.

In order to increase the E.E. and the loading capacity of PEG-NC, we applied a simple modification of the solvent displacement technique that consists on buffering the continuous aqueous phase used for nanocarrier preparation at $\mathrm{pH} 9$ with sodium carbonate (buffer concentration $10 \mathrm{mM}$ ) [9, 21]. The resulting E.E. value was $85 \%$ for a drug concentration of $2 \mathrm{mg} / \mathrm{mL}$. This change implied a 7 -fold increase, both in E.E. and real drug loading as compared to the non-modified solvent displacement technique. Interestingly, similarly high E.E. $(\approx 90 \%)$ could be achieved with the standard PEG-NC if working at lower drug concentration $(50 \mu \mathrm{g} / \mathrm{mL})$.

Table 2 shows the physicochemical characterization of blank and drug-loaded PEG-NC prepared at different $\mathrm{pH}$ values: 7 and 9. It can be seen that, at any $\mathrm{pH}$, the size of both PEG-NC increases after drug encapsulation, from $175 \mathrm{~nm}$ to $250 \mathrm{~nm}$; this is probably due to the incorporation of significant amounts of drug to the nanocarriers. Analysis of PEG-NC surface charge by Z-potential shows significant differences between the two formulations. Blank PEG-NC showed negative Z-potential values at both $\mathrm{pH}$ values 
(both around $-20 \mathrm{mV}$ ), caused mainly by the presence of phospholipids on their surface, which remain negatively charged at any $\mathrm{pH}$. However, the Z-potential of PEG-NC prepared at $\mathrm{pH} 7$ is dramatically inverted upon drug encapsulation, going from $-20 \mathrm{mV}$ to $+38 \mathrm{mV}$. This inversion of the Z-potential could only be attributed to the presence of positively charged drug on the PEG-NC surface. Interestingly, this inversion in the Z-potential value was not observed for PEG-NC prepared at $\mathrm{pH}$ 9, even at this high drug concentrations.

This difference in behavior regarding E.E. and Z-potential of PEG-NC prepared at $\mathrm{pH} 7$ and $\mathrm{pH} 9$ can be explained by two non-excluding hypothesis. First, by deprotonation of the drug on the PEG-NC surface at $\mathrm{pH}$ 9. Another explanation would be that deprotonated F10320D1 becomes more affine for the oil, and that this change results in its preferential allocation in the nanocarrier core (Scheme 1). Further studies suggest that this last hypothesis should account for most of the observed effect (see 3.3 and 3.4).

\subsection{Stability studies with drug-loaded formulations.}

Drug-loaded PEG-NC were stored at $4{ }^{\circ} \mathrm{C}$ for 1 month and size, P.I., drug concentration and E.E. were measured at different time points to evaluate the stability of the selected formulations. Size and P.I. remained unchanged for PEG-NC prepared at $\mathrm{pH} 7$ and $\mathrm{pH}$ 9: size $\approx 250 \mathrm{~nm}$ and P.I. $\approx 0.15$ (Supplementary Figure S2). These results indicate adequate colloidal stability of the nanocarriers when stored at $4^{\circ} \mathrm{C}$ for prolonged periods. The stability of the drug-loaded PEG-NC in terms of size and zeta potential was similar to that of blank PEG-NC, which indicates that the presence of drug does not affect the physicochemical stability of the PEG-NC.

A different situation was observed when we analyzed the formulations drug content over time (Figure 4). PEG-NC prepared at $\mathrm{pH} 7$ presented a dramatic diminution (80\%) of encapsulated drug already after 15 days of storage. In contrast, PEG-NC prepared at $\mathrm{pH} 9$ showed no drug leakage after 1 month. Total drug concentration (encapsulated plus non-encapsulated) was also found to remain constant during the stability studies for PEG-NC prepared at pH 9, but not for those prepared at $\mathrm{pH} 7$ (Supplementary information, Figure S3). The improved stability of PEG-NC formulated at $\mathrm{pH} 9$ in comparison with those formulated at $\mathrm{pH} 7$, could be explained by the efficient drug encapsulation within the inert oily core instead of being simply associated on the nanocarrier surface.

\section{4 in vitro drug release}

In vitro drug release studies were performed under sink conditions in PBS at $37^{\circ} \mathrm{C}$ (Figure 5). PEG-NC loaded with F10320GD1 and prepared at pH 7 and 9 were studied for release for a period of 8 hours. 
PEG-NC prepared at $\mathrm{pH} 7$ presented an important burst release of around 50\% of the drug payload, followed by a plateau phase for the next 8 hours. This drug release profile is typical for most oily-based formulations such as polymer-coated nanocapsules [33, 37-39]. For PEG-NC formulated at $\mathrm{pH} 9$, the burst effect was significantly reduced from $50 \%$ to $15 \%$, and the amount of drug released after $8 \mathrm{~h}$ was still only $30 \%$ of the payload. Therefore, PEG-NC prepared at $\mathrm{pH} 9$ showed a controlled release profile, in contrast with the uncontrolled behavior of PEG-NC prepared at $\mathrm{pH}$ 7. Sustained release profiles have been also observed from some lipid nanocarriers upon deprotonation of doxorubicin [40] and etoposide [27].

This difference in drug release profile is particularly remarkable because once in PBS all formulations are at the same $\mathrm{pH}$, and therefore, partition and solubility equilibria should be equal for both PEG-NC independently of their $\mathrm{pH}$ preparation value. Thus, the more sustained drug release profile observed for PEG$\mathrm{NC}$ prepared at $\mathrm{pH} 9$ should be related to the drug allocation within the oily core, and the necessity to overcome a diffusional barrier to participate in solubility/partition equilibria. The diffusional barrier could be the phospholipid/PEG-STE shell as previous studies have shown that PEG-STE is preferentially disposed on the surface of lipid nanocarriers [16], and that this material can form packed lipid layers with a melting point between 40 and $51^{\circ} \mathrm{C}$ depending on its physical form [19]. On this basis, a lecithin/PEGSTE lipid layer could be an effective barrier to control drug release, providing that the drug is integrated in the nanocarrier's core.

\subsection{In vitro antitumor efficacy studies.}

Finally, the bioactivity of F10320GD1 loaded into PEG-NC was assessed in different cell models. Cytotoxicity of F10320GD1 dissolved in 2\% DMSO, drug loaded PEG-NC (300 $\mu \mathrm{g} / \mathrm{mL})$ and blank PEG-NC was evaluated in three different human tumor cell lines: MCF-7, NCI-H460 and SF-268 (from lung, breast and glioma, respectively). The formulations were also tested in human umbilical vein endothelial cells (HUVEC) to asses for differential toxicity in non-tumor cells.

In all the tumor cell lines, there was a significant increase of the cytotoxic effect when the drug was encapsulated on PEG-NC as compared to the drug in solution (Figure 6). For instance, the IC50 in MCF-7 cell line for non-encapsulated drug was $6.5 \mu \mathrm{M}$, while for the drug loaded in PEG-NC was $2.8 \mu \mathrm{M}$, a two-fold increase in efficacy. Similar results were obtained in NCI-H460, where PEG-NC delivery reduced IC50 values from $16 \mu \mathrm{M}$ to $9.5 \mu \mathrm{M}$. In SF-268, IC50 was reduced from $7.8 \mu \mathrm{M}$ to $2.8 \mu \mathrm{M}$. The IC50 values obtained for blank PEG-NC were 20-30 $\mu \mathrm{M}$, depending on the cell line. This IC50 values are expressed as the corresponding drug concentration if blank PEG-NC would have been loaded with 
F10320GD1; these values are over 2-3 times higher than the drug alone, and they indicates that the formulation should not be a relevant contributor to the toxic effect observed in drug-loaded samples per se. The increase in drug cytotoxicity when encapsulated in PEG-NC has previously been observed by other authors. For instance, the group of Benoit et al. observed a significant enhancement of the therapeutic effect of several drugs formulated in PEG-NC such as etoposide [27], tamoxifen [28], or paclitaxel [29]. In addition, Mumper et al. have also reported a decrease on the IC50 values of paclitaxel and doxorubicin after encapsulation in PEG-NC [11]. Having discarded a direct toxic effect of PEG-NC, the higher efficacy of the drugs upon encapsulation in PEG-NC can be attributed to two effects: (a) the easier internalization of the loaded PEG-NC into the cytosol as compared to the drug alone [27-28], or/and (b) the possible inhibition of the efflux mechanisms of the cancer cells due to the presence of PEG polymers or by the nanocapsules themselves [41-42]. In fact, it has been widely reported that poloxamer inhibits many cell's efflux pumps, such as $\mathrm{P}-\mathrm{gP}$ or MDR proteins, through different pathways [43].

Cytotoxicity of F10320GD1 alone or formulated in PEG-NC as well as the PEG-NC alone was also tested in HUVEC, to check for tumor/non-tumor differential toxicity. The results showed a significant decrease of the IC50 values for loaded PEG-NC, but this decrease is unnoticeable as compared to that observed in tumor cell lines. In addition, no cytotoxic effect was observed for blank PEG-NC in HUVEC cells, which confirms the low toxicity profile of this carrier over the studied concentrations.

\section{4- CONCLUSIONS}

Herein we report on the nanoencapsulation of a challenging compound, with limited solubility in water and in oils, in PEGylated nanocapsules. High drug encapsulation efficacy with high drug payloads were achieved by controlling the $\mathrm{pH}$ of the continuous phase during the nanoencapsulation process. Besides, this control in the $\mathrm{pH}$ also resulted in other beneficial characteristics such as prolonged drug stability upon storage, and controlled release properties. The data obtained suggest that these improved properties might be linked to a change in drug allocation to the inner core of the nanocapsules caused by $\mathrm{pH}$-dependent deprotonation. In vitro testing in a cell culture panel indicated that the drug formulated in PEG-NC is bioactive, and even more efficacious than the drug itself, indicating improved intracellular delivery of the nanoencapsulated drug. We believe that the technological approach outlined in this work could be valuable for formulating other similar compounds in structured lipid nanocarriers.

\section{LIST OF ABBREVIATIONS:}

D-MEM, Dulbecco's Modified Eagle Medium, DMSO, dimethyl sulfoxide, E.E. encapsulation efficiency, HPLC, high performance liquid chromatography, HNMR, proton nuclear magnetic reso- 
nance, HUVEC, human umbilical vein endothelial cells, IC50, 50\% inhibiton concentration, i.v. intravenous, LDA, laser Doppler anemometry, PBS, phosphate buffered saline, PCS, photon correlation spectroscopy, PEG-NC, Pegylated Nanocapsules, PEG-STE, Polyethyleneglycol steareate, P.I. polydispersity index, XTT, 2,3-Bis-(2-Methoxy-4-Nitro-5-Sulfophenyl)-2H-Tetrazolium-5-Carboxanilide.

\section{CONFLICT OF INTERST:}

Francisco Ledo and Maria Luisa Lucero are employees of FAES Farma, S.A. F10320GD1 is a patented drug candidate from FAES Farma, S.A. The authors declare no other competing conflict of interest

\section{ACKNOWLEDGEMENTS}

The authors would like to acknowledge financial support from CENIT-NANOFAR XS53 project, FAES Farma S.A. (Spain), Xunta de Galicia (Competitive Reference Ref. GRC2014/043, FEDER Funds) and the European Commission FP7 EraNet - EuroNanoMed Program-Instituto Carlos III (Lymphotarg proyect, Ref. PS09/02670). MGF was a recipient of an Isidro Parga Pondal contract. 
REFERENCES

[1] Hervella, P.; Lozano, V.; Garcia-Fuentes, M.; Alonso, M. J., Nanomedicine: New Challenges and Opportunities in Cancer Therapy. J. Biomed. Nanotechnol., 2008, 4 (3), 276-292.

[2] Mura, S.; Couvreur, P., Nanotheranostics for personalized medicine. Adv. Drug Deliver. Rev., 2012, 64 (13), 1394-1416.

[3] Owens, D. E.; Peppas, N. A., Opsonization, biodistribution, and pharmacokinetics of polymeric nanoparticles. Int. J. Pharm., 2006, 307 (1), 93-102.

[4] Torchilin, V. P., Multifunctional nanocarriers. Adv. Drug Deliver. Rev., 2006, 58 (14), 1532-1555.

[5] Wang, A. Z.; Langer, R.; Farokhzad, O. C., In Annual Review of Medicine, Caskey, C. T.; Austin, C. P.; Hoxie, J. A., Eds. 2012; Vol. 63, pp 185-198.

[6] Feng, L.; Mumper, R. J., A critical review of lipid-based nanoparticles for taxane delivery. Cancer Lett., 2013, 334 (2), 157-175.

[7] Mora-Huertas, C. E.; Fessi, H.; Elaissari, A., Polymer-based nanocapsules for drug delivery. Int. J. Pharm., 2010, 385 (1-2), 113-142.

[8] Siepmann, J.; Kranz, H.; Peppas, N. A.; Bodmeier, R., Calculation of the required size and shape of hydroxypropyl methylcellulose matrices to achieve desired drug release profiles. Int. J. Pharm., 2000, 201 (2), 151-164.

[9] Kataoka, K.; Matsumoto, T.; Yokoyama, M.; Okano, T.; Sakurai, Y.; Fukushima, S.; Okamoto, K.; Kwon, G. S., Doxorubicin-loaded poly(ethylene glycol)-poly(betabenzyl-l-aspartate) copolymer micelles: their pharmaceutical characteristics and biological significance. J. Control. Release, 2000, 64 (1-3), 143-153.

[10] Tewes, F.; Munnier, E.; Antoon, B.; Okassa, L. N.; Cohen-Jonathan, S.; Marchais, H.; Douziech-Eyrolles, L.; Souce, M.; Dubois, P.; Chourpa, I., Comparative study of doxorubicin-loaded poly(lactide-co-glycolide) nanoparticles prepared by single and double emulsion methods. Eur. J. Pharm. Biopharm., 2007, 66 (3), 488-492.

[11] Dong, X.; Mattingly, C. A.; Tseng, M. T.; Cho, M. J.; Liu, Y.; Adams, V. R.; Mumper, R. J., Doxorubicin and Paclitaxel-Loaded Lipid-Based Nanoparticles Overcome Multidrug Resistance by Inhibiting P-Glycoprotein and Depleting ATP. Cancer Res., 2009, 69 (9), 3918-3926. 
[12] Vrignaud, S.; Anton, N.; Gayet, P.; Benoit, J.-P.; Saulnier, P., Reverse micelleloaded lipid nanocarriers: A novel drug delivery system for the sustained release of doxorubicin hydrochloride. Eur. J. Pharm. Biopharm., 2011, 79 (1), 197-204.

[13] Mora-Huertas, C. E.; Garrigues, O.; Fessi, H.; Elaissari, A., Nanocapsules prepared via nanoprecipitation and emulsification-diffusion methods: Comparative study. Eur. J. Pharm. Biopharm., 2012, 80 (1), 235-239.

[14] Beduneau, A.; Saulnier, P.; Hindre, F.; Clavreul, A.; Leroux, J.-C.; Benoit, J.-P., Design of targeted lipid nanocapsules by conjugation of whole antibodies and antibody Fab' fragments. Biomaterials, 2007, 28 (33), 4978-4990.

[15] Diaz-Lopez, R.; Tsapis, N.; Santin, M.; Bridal, S. L.; Nicolas, V.; Jaillard, D.; Libong, D.; Chaminade, P.; Marsaud, V.; Vauthier, C.; Fattal, E., The performance of PEGylated nanocapsules of perfluorooctyl bromide as an ultrasound contrast agent. Biomaterials, 2010, 31 (7), 1723-1731.

[16] Garcia-Fuentes, M.; Torres, D.; Martín-Pastor, M.; Alonso, M. J., Application of NMR Spectroscopy to the Characterization of PEG-Stabilized Lipid Nanoparticles. Langmuir, 2004, 20 (20), 8839-8845.

[17] Needham, D.; McIntosh, T. J.; Lasic, D. D., Repulsive interactions and mechanical stability of polymer-grafted lipid membranes. Biochim. Biophys. Acta, 1992, 1108 (1), 40-48.

[18] Needham, D.; Kim, D., PEG-covered lipid surfaces: bilayers and monolayers. Colloid. Surface. B, 2000, 18 (3-4), 183-195.

[19] Garcia-Fuentes, M.; Alonso, M. J.; Torres, D., Design and characterization of a new drug nanocarrier made from solid-liquid lipid mixtures. J. Colloid Interf. Sc., 2005, 285 (2), 590-598.

[20] Haran, G.; Cohen, R.; Bar, L. K.; Barenholz, Y., Transmembrane ammonium sulfate gradients in liposomes produce efficient and stable entrapment of amphipathic weak bases. Biochim. Biophys. Acta, 1993, 1151 (2), 201-215.

[21] Sanson, C.; Schatz, C.; Le Meins, J. F.; Soum, A.; Thevenot, J.; Garanger, E.; Lecommandoux, S., A simple method to achieve high doxorubicin loading in biodegradable polymersomes. J. Control. Release, 2010, 147 (3), 428-435.

[22] Calvo, P.; Vila-Jato, J. L.; Alonso, M. J., Comparative in vitro evaluation of several colloidal systems, nanoparticles, nanocapsules, and nanoemulsions, as ocular drug carriers. J. Pharm. Sci., 1996, 85 (5), 530-6.

[23] Mollinedo, F.; Gajate, C.; Morales, A. I.; del Canto-Jañez, E.; Justies, N.; Collía, F.; Rivas, J. V.; Modolell, M.; Iglesias, A., Novel Anti-Inflammatory Action of Edelfosine Lacking Toxicity with Protective Effect in Experimental Colitis. J. Pharmacol. Exp. Ther., 2009, 329 (2), 439-449. 
[24] Constantinides, P. P.; Chaubal, M. V.; Shorr, R., Advances in lipid nanodispersions for parenteral drug delivery and targeting. Adv. Drug Deliver. Rev., 2008, 60 (6), 757-767.

[25] Kelmann, R. G.; Kuminek, G.; Teixeira, H. F.; Koester, L. S., Carbamazepine parenteral nanoemulsions prepared by spontaneous emulsification process. Int. J. Pharm., 2007, 342 (1-2), 231-239.

[26] Garcia-Fuentes, M.; Torres, D.; Alonso, M. J., Design of lipid nanoparticles for the oral delivery of hydrophilic macromolecules. Colloid. Surface. B, 2003, 27 (2-3), 159-168.

[27] David, S.; Carmoy, N.; Resnier, P.; Denis, C.; Misery, L.; Pitard, B.; Benoit, J.P.; Passirani, C.; Montier, T., In vivo imaging of DNA lipid nanocapsules after systemic administration in a melanoma mouse model. Int. J. Pharm., 2012, 423 (1), 108-115.

[28] Huynh, N. T.; Passirani, C.; Allard-Vannier, E.; Lemaire, L.; Roux, J.; Garcion, E.; Vessieres, A.; Benoit, J.-P., Administration-dependent efficacy of ferrociphenol lipid nanocapsules for the treatment of intracranial 9L rat gliosarcoma. Int. J. Pharm., 2012, 423 (1), 55-62.

[29] Huynh, N. T.; Passirani, C.; Saulnier, P.; Benoit, J. P., Lipid nanocapsules: A new platform for nanomedicine. Int. J. Pharm., 2009, 379 (2), 201-209.

[30] Weiszhár, Z.; Czúcz, J.; Révész, C.; Rosivall, L.; Szebeni, J.; Rozsnyay, Z., Complement activation by polyethoxylated pharmaceutical surfactants: Cremophor-EL, Tween-80 and Tween-20. Eur. J. Pharm. Sci., 2012, 45 (4), 492-498.

[31] d'Angelo, I.; Garcia-Fuentes, M.; Parajo, Y.; Welle, A.; Vantus, T.; Horvath, A.; Boekoenyi, G.; Keri, G.; Jose Alonso, M., Nanoparticles Based on PLGA:Poloxamer Blends for the Delivery of Proangiogenic Growth Factors. Mol. Pharm., 2010, 7 (5), 1724-1733.

[32] Trapani, A.; Lopedota, A.; Franco, M.; Cioffi, N.; Ieva, E.; Garcia-Fuentes, M.; Jose Alonso, M., A comparative study of chitosan and chitosan/cyclodextrin nanoparticles as potential carriers for the oral delivery of small peptides. Eur. J. Pharm. Biopharm., 2010, 75 (1), 26-32.

[33] Lozano, M. V.; Torrecilla, D.; Torres, D.; Vidal, A.; Dominguez, F.; Alonso, M. J., Highly efficient system to deliver taxanes into tumor cells: docetaxel-loaded chitosan oligomer colloidal carriers. Biomacromolecules, 2008, 9 (8), 2186-93.

[34] de Faria, T. J.; Machado de Campos, A.; Lemos Senna, E., Preparation and Characterization of Poly(D,L-Lactide) (PLA) and Poly(D,L-Lactide)-Poly(Ethylene Glycol) (PLA-PEG) Nanocapsules Containing Antitumoral Agent Methotrexate. Macromol. Symp., 2005, 229 (1), 228-233. 
[35] zur Mühlen, A.; Schwarz, C.; Mehnert, W., Solid lipid nanoparticles (SLN) for controlled drug delivery - Drug release and release mechanism. Eur. J. Pharm. Biopharm., 1998, 45 (2), 149-155.

[36] Maeda, H.; Wu, J.; Sawa, T.; Matsumura, Y.; Hori, K., Tumor vascular permeability and the EPR effect in macromolecular therapeutics: a review. J. Control. Release, 2000, 65 (1-2), 271-284.

[37] Prego, C.; Torres, D.; Fernandez-Megia, E.; Novoa-Carballal, R.; Quinoa, E.; Alonso, M. J., Chitosan-PEG nanocapsules as new carriers for oral peptide delivery. Effect of chitosan pegylation degree. J Control Release, 2006, 111 (3), 299-308.

[38] Lozano, M. V.; Lollo, G.; Alonso-Nocelo, M.; Brea, J.; Vidal, A.; Torres, D.; Alonso, M. J., Polyarginine nanocapsules: a new platform for intracellular drug delivery. J. Nanopart. Res., 2013, 15 (3), 1-14.

[39] Gonzalo, T.; Lollo, G.; Garcia-Fuentes, M.; Torres, D.; Correa, J.; Riguera, R.; Fernandez-Megia, E.; Calvo, P.; Aviles, P.; Guillen, M. J.; Alonso, M. J., A new potential nano-oncological therapy based on polyamino acid nanocapsules. J. Control. Release, 2013, 169 (1-2), 10-16.

[40] David, S.; Resnier, P.; Guillot, A.; Pitard, B.; Benoit, J.-P.; Passirani, C., siRNA LNCs - A novel platform of lipid nanocapsules for systemic siRNA administration. Eur. J. Pharm. Biopharm., 2012, 81 (2), 448-452.

[41] Lamprecht, A.; Benoit, J. P., Etoposide nanocarriers suppress glioma cell growth by intracellular drug delivery and simultaneous P-glycoprotein inhibiltion. J. Control. Release, 2006, 112 (2), 208-213.

[42] Ma, P.; Mumper, R. J., Anthracycline nano-delivery systems to overcome multiple drug resistance: A comprehensive review. Nano Today, 2013, 8 (3), 313-331.

[43] Kabanov, A. V.; Batrakova, E. V.; Alakhov, V. Y., Pluronic((R)) block copolymers for overcoming drug resistance in cancer. Adv. Drug Deliver. Rev., 2002, 54 (5), 759-779. 


\section{TABLES}

Table 1. Effect of PEGylation on size, polydispersity index (P.I.), and Zeta potential of PEG-NC. Data are means \pm S.D., $\mathrm{n}=4$.

\begin{tabular}{cccc} 
\% PEG-STE w/w & Size $(\mathrm{nm})$ & P.I. & Z-potential $(\mathrm{mV})$ \\
\hline 0 & $184 \pm 2$ & 0.13 & $-38 \pm 2$ \\
15 & $175 \pm 7$ & 0.12 & $-34 \pm 2$ \\
30 & $176 \pm 13$ & 0.13 & $-22 \pm 2$ \\
60 & $178 \pm 21$ & 0.12 & $-28 \pm 2$
\end{tabular}

Table 2. Mean particle size distribution, polydispersity index (P.I. ), Zeta potential and Encapsulation efficiency (E.E.) of PEG-NC prepared with two different continuous phases: buffer $\mathrm{pH} 7$ and buffer $\mathrm{pH}$ 9. Data are means \pm S.D., $n=4$.

\begin{tabular}{ccccccc} 
Formulation & $\mathrm{pH}$ & $\begin{array}{c}\text { Size } \\
(\mathrm{nm})\end{array}$ & P.I. & $\begin{array}{c}\text { Z-potential } \\
(\mathrm{mV})\end{array}$ & $\begin{array}{c}{[\mathrm{F} 10320 \mathrm{GD} 1]} \\
(\mathrm{mg} / \mathrm{mL})\end{array}$ & E.E. (\%) \\
\hline PEG-NC (blank) & 7 & $176 \pm 5$ & 0.13 & -22 & 0 & $/$ \\
PEG-NC (blank) & 9 & $176 \pm 5$ & 0.15 & -16 & 0 & $15 \pm 5$ \\
PEG-NC (loaded) & 7 & $239 \pm 4$ & 0.22 & +38 & 2 & $85 \pm 10$ \\
PEG-NC (loaded) & 9 & $241 \pm 3$ & 0.23 & -12 & 2 &
\end{tabular}




\section{FIGURES}

Scheme 1 Schematic illustration of the solvent displacement techniques described in this work for the formation of PEG-NC.
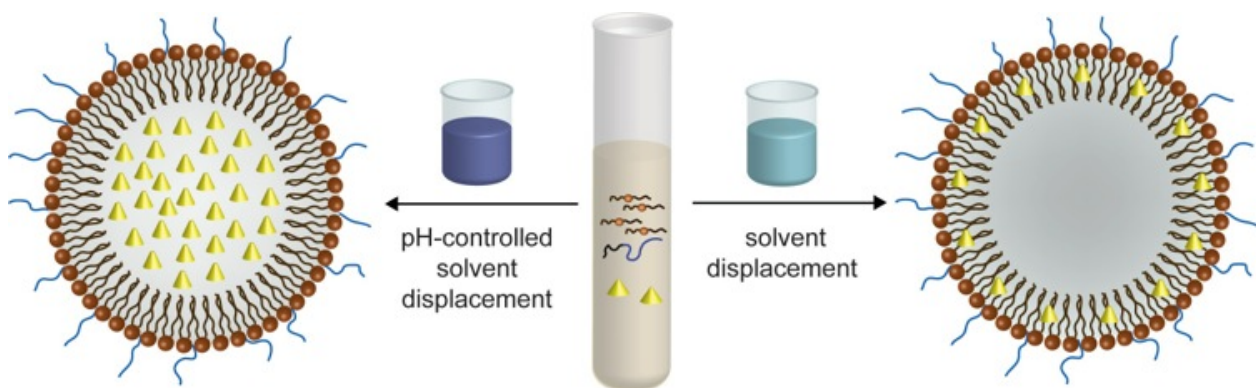

High loading capacity. High stability. Sustained release.

$\triangle$ Drug (F10320GD1)

Phospholipids (Soybean lecithin)

2 PEG-Stearate
Low loading capacity.

Low stability.

Fast release.

Table 1. Effect of PEGylation over size, P.I. and Zeta potential of PEG-NC. Data are means \pm S.D., $n=4$

\begin{tabular}{cccc} 
\% PEG-STE w/w & Size $(\mathrm{nm})$ & P.I. & Z-potential $(\mathrm{mV})$ \\
\hline 0 & $184 \pm 2$ & 0.13 & $-38 \pm 2$ \\
15 & $175 \pm 7$ & 0.12 & $-34 \pm 2$ \\
30 & $176 \pm 13$ & 0.13 & $-22 \pm 2$ \\
60 & $178 \pm 21$ & 0.12 & $-28 \pm 2$
\end{tabular}

Table 2. Size, P.I. Zeta potential and Encapsulation efficiency (E.E.) of blank and loaded PEG-NC prepared in two different buffers at $\mathrm{pH} 7$ and 9. Data are means \pm S.D., $\mathrm{n}=4$

\begin{tabular}{ccccccc} 
Formulation & $\mathrm{pH}$ & Size & P.I. & $\begin{array}{c}\text { Z-potential } \\
(\mathrm{mm})\end{array}$ & $\begin{array}{c}\text { [F10320GD1] } \\
(\mathrm{mg} / \mathrm{mL})\end{array}$ & E.E. $(\%)$ \\
\hline blank-PEG-NC & 7 & $176 \pm 5$ & 0.13 & -22 & 0 & $/$ \\
blank-PEG-NC & 9 & $176 \pm 5$ & 0.15 & -16 & 0 & $/$ \\
Loaded-PEG-NC & 7 & $239 \pm 4$ & 0.22 & +38 & 2 & $25 \pm 5$ \\
Loaded-PEG-NC & 9 & $241 \pm 3$ & 0.23 & -12 & 2 & $85 \pm 10$
\end{tabular}


Figure 1. TEM images of blank PEG-NC prepared with different amounts of PEG-STE (\% w/w): 0 (a), 30 (b) and 60 (c). Scale bar $=200 \mathrm{~nm}$.

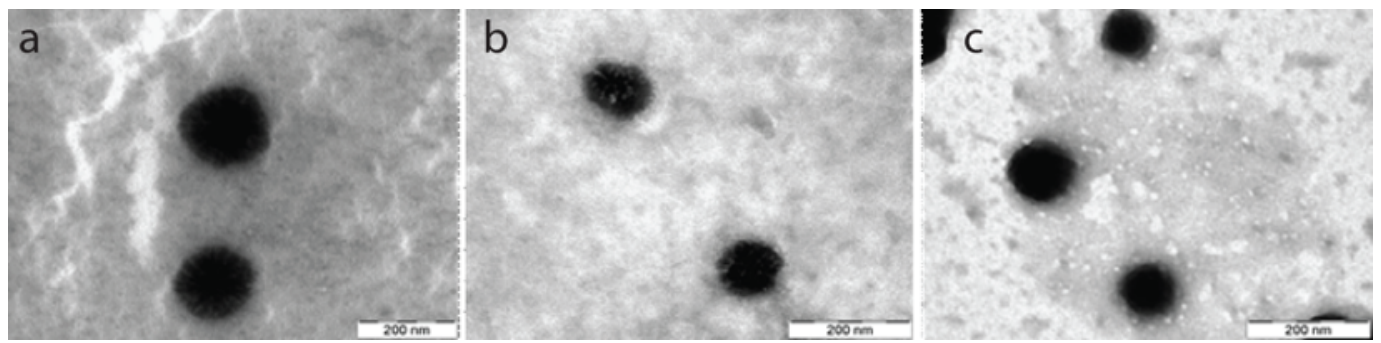

Figure 2. Size of PEG-NC in presence or in the absence of poloxamer, after 24 hours incubation at $37^{\circ} \mathrm{C}$ in different media: water (control), saline solution $0.9 \%$, supplemented medium (D-MEM+) and phosphate buffer (PBS). Data represents means \pm S.D., $n=4$.

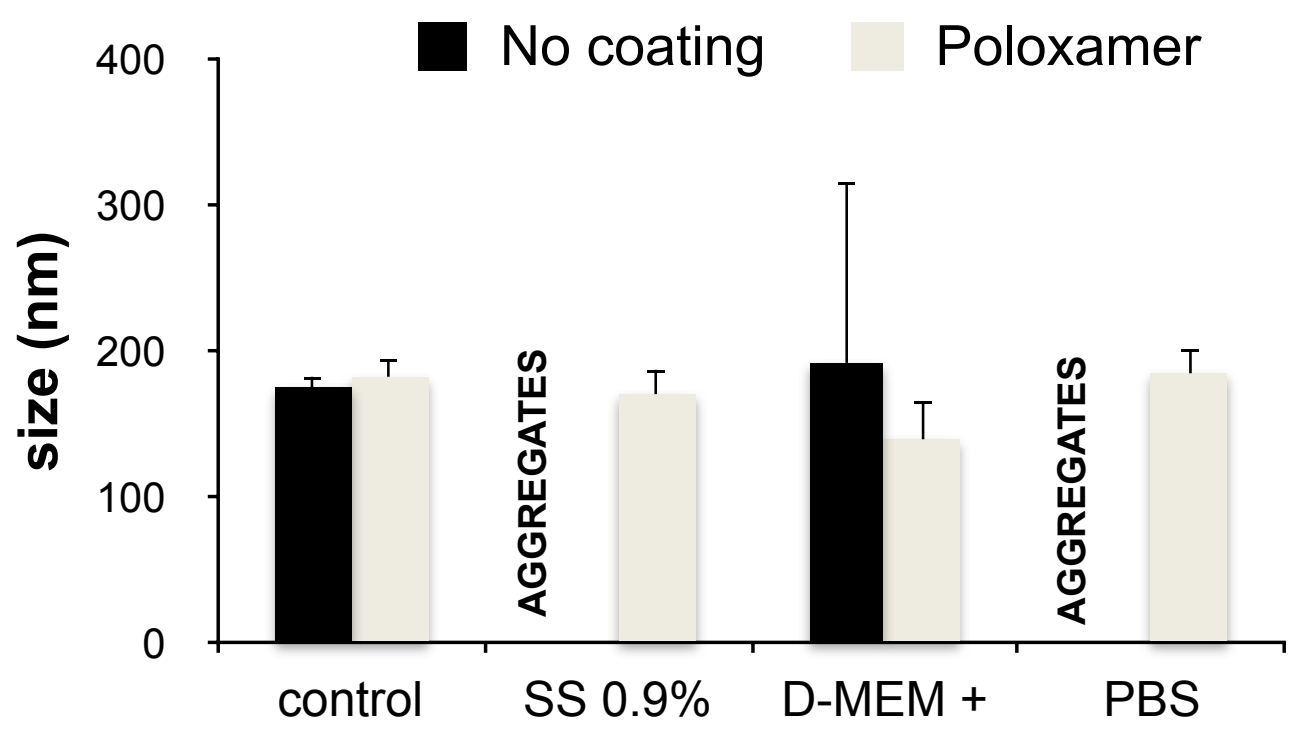


Figure 3. Size of blank PEG-NCs upon storage at $4{ }^{\circ} \mathrm{C}, 25^{\circ} \mathrm{C}$ and $37^{\circ} \mathrm{C}$. Polydispersity index remained below 0.15 in all cases. means \pm S.D., $n=4$.

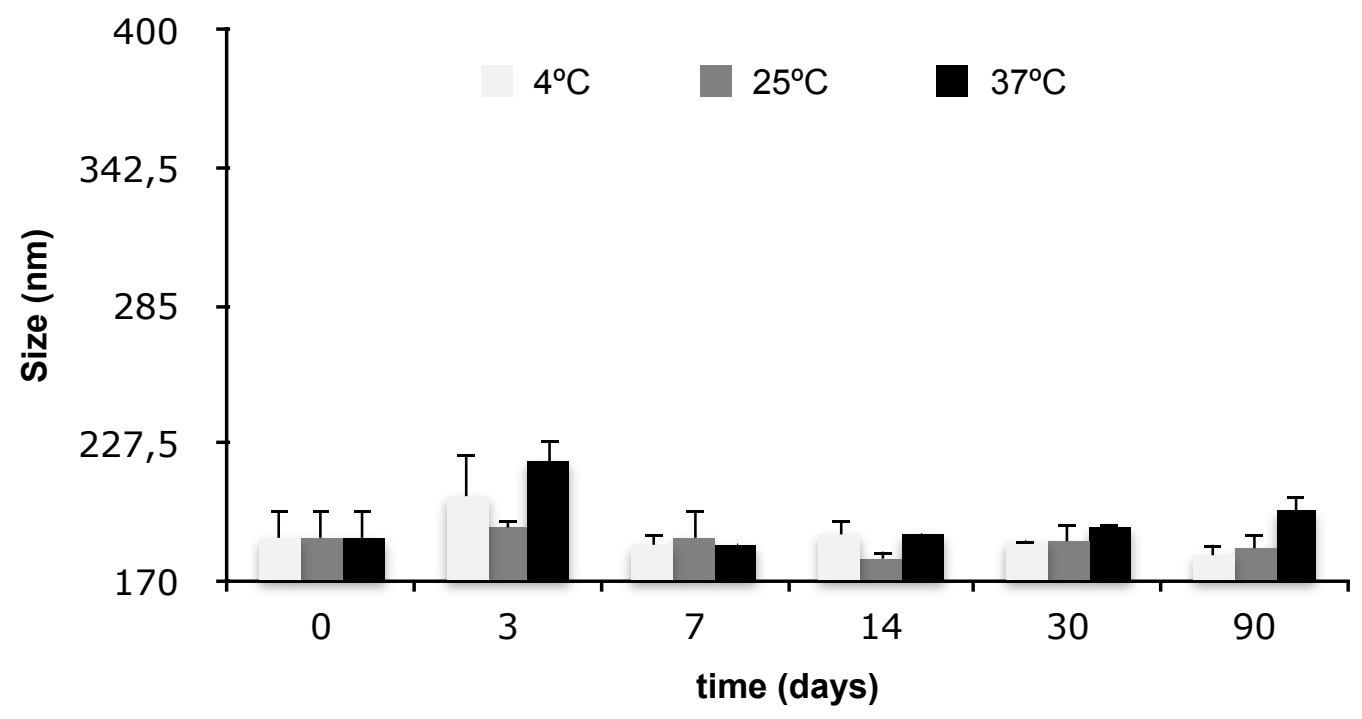

Figure 4. Variation of E.E. of loaded PEG-NC prepared at $\mathrm{pH} 7$ and $\mathrm{pH} 9$ under storage at $4^{\circ} \mathrm{C}$. Initial drug concentration was $0.05 \mathrm{mg} / \mathrm{mL}$ for PEG-NC prepared at $\mathrm{pH} 7$ and $2 \mathrm{mg} / \mathrm{mL}$ for PEG-NC prepared at $\mathrm{pH}$ 9. Data represents means \pm S.D., $\mathrm{n}=4$.

$\mathrm{pH} 7$

$\mathrm{pH} 9$

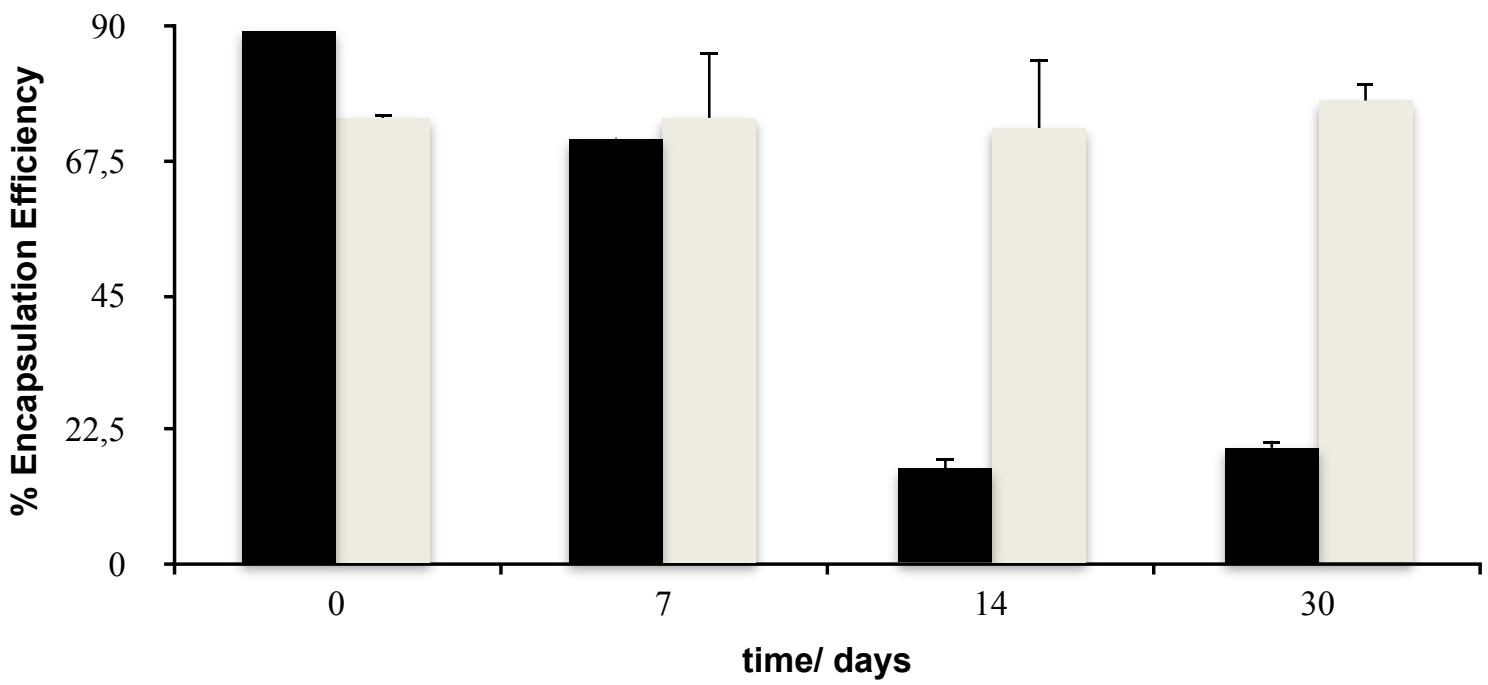


Figure 5. In vitro drug release profiles of PEG-NC prepared at $\mathrm{pH} 7$ (black circles) and $\mathrm{pH} 9$ (white squares) and incubated in $\mathrm{PBS}(\mathrm{pH}=7.4)$ at $37^{\circ} \mathrm{C}$. Stars indicate the significant difference of data points compared with the previous one $(* \mathrm{p}<0.05)$. Data represents means \pm S.D., $n=4$.
- $\mathrm{pH} 7$
$\mathrm{pH} 9$

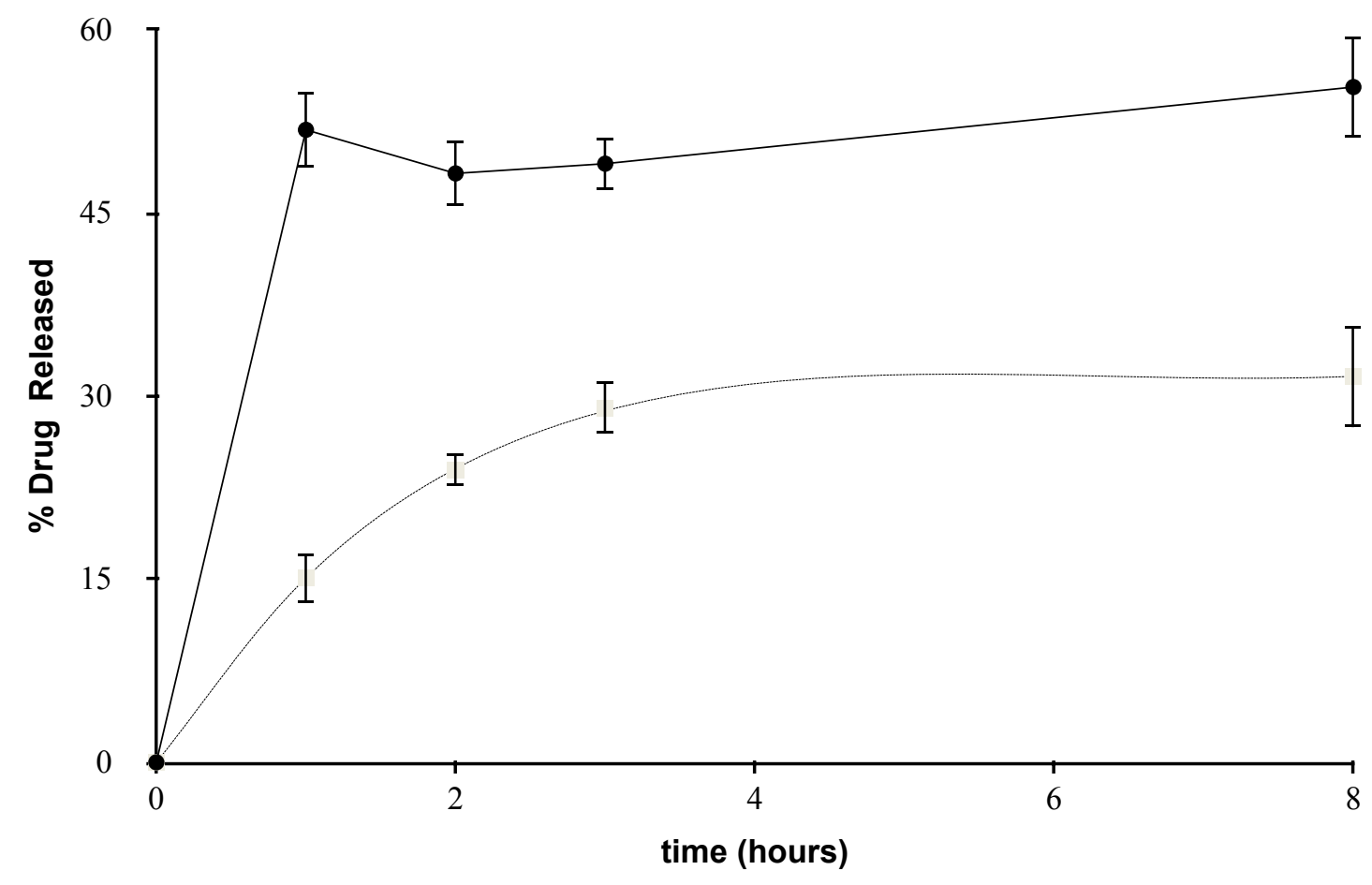


Figure 6. Inhibitory concentration 50\% (IC50) values obtained for the free drug (F10320GD1), loaded PEG-NC (loaded) and blank PEG-NC (blank) in: MCF-7, NCI-H460 and SF-268 tumor cell lines, and in Human Umbilical Vein Endothelial Cells (HUVEC) primary cultures. Data represents means \pm S.D. $n=12$, four independent experiments each performed in triplicates. $(* \mathrm{p}<0.05, * * \mathrm{p}<0.01, * * * \mathrm{p}<0.005)$.

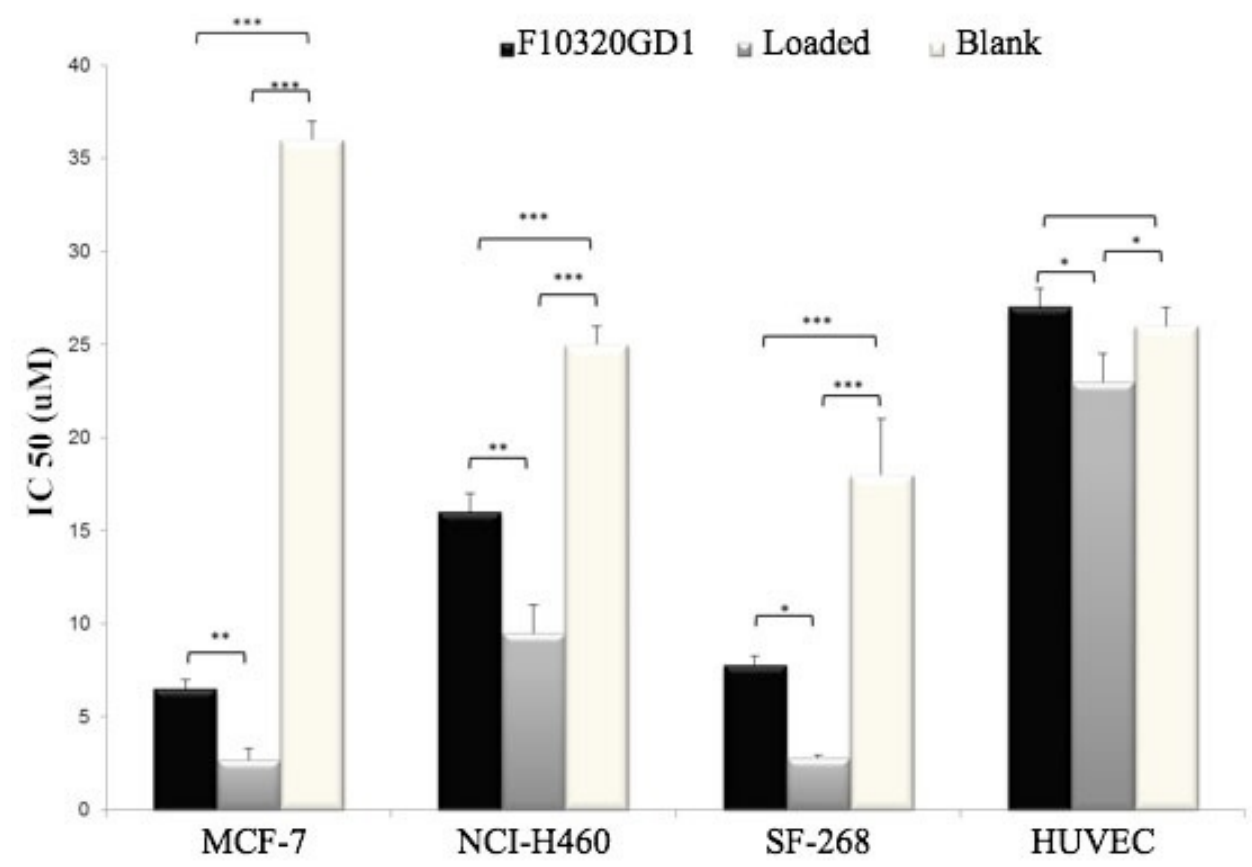

